\title{
Selection of a Trajectory Function for Minimum Energy Requirements of a Spherical Robot
}

\author{
A. A. N. Aljawi, H. Diken AND S. A. Alshahrani \\ Department of Production Engineering and Mechanical Systems Design, \\ Faculty of Engineering, King Abdulaziz University, \\ Jeddah, Saudi Arabia.
}

Corresponding author: hdiken@yahoo.com

\begin{abstract}
In this study dynamic equations of a Stanford type spherical robot is developed. In the work space of the robot, straight line trajectory is chosen for the end effector. On this trajectory, robot travels with different time functions. These functions are cubic segment, bang-bang parabolic blend, simple harmonic, cycloid, and 3-4-5 polynomial. Total consumed energy curves are obtained with respect to travel times for each trajectory. Results show that cubic segment trajectory function spends minimum energy.
\end{abstract}

Keywords: manipulator dynamics, trajectory planning.

\section{Analysis}

The Stanford type spherical robot is shown in Fig. 1. Equations of motion are derived considering only the body, back arm and the forearm. The second set of three motions for the manipulator hand, which gives orientation to the payload, are ignored. The Denavit-Hartenberg method is used to find forward and inverse kinematic relations ${ }^{[1]}$. Total kinetic energy of the robot is then obtained by using kinematic relations and dynamic parameters.

When Lagrange equations are applied, the following equations of motion, in matrix form are obtained. 


$$
D(\theta) \ddot{\theta}+N\left(\dot{\theta}^{2}\right)+C\left(\dot{\theta}_{i} \dot{\theta}_{j}\right)+G(\theta)=T
$$

Generalized coordinate vector is $\theta=\left[\begin{array}{ll}\theta_{1} & \theta_{2}\end{array}\right]^{T}$, here $\theta_{1}$ and $\theta_{2}$ are rotations $r$ is translation Torque vector is $T=\left[\begin{array}{lll}\tau_{1} & \tau_{2} f\end{array}\right]^{T}$, here $\tau_{1}$ and $\tau_{2}$ are motor torques $f$ is the linear force. $D$ is the inertia matrix, $N$ is the normal force matrix, $C$ is the Coriolis force matrix, $G$ is the gravitational force matrix and $T$ is the torque vector, respectively.

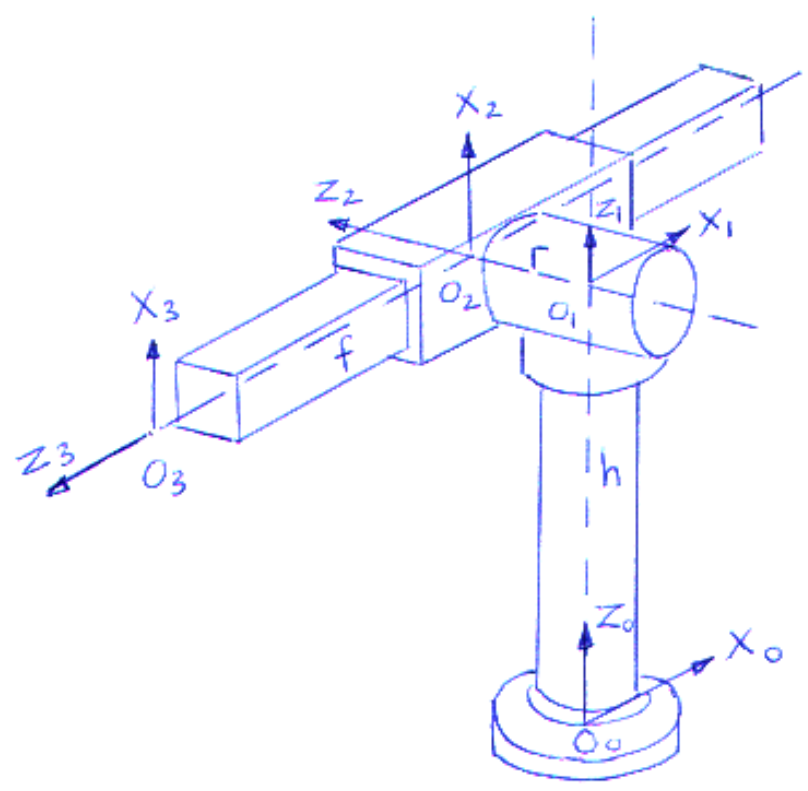

Fig. 1. Stanford type spherical manipulator.

\section{Trajectories}

It is assumed that the end point of the manipulator travels from one point to another in Cartesian space on a straight line, which can be given as,

$$
P=P_{0}+\lambda(t)\left\lfloor P_{f}-P_{0}\right\rfloor
$$

$\lambda(t)$ is the time function, such that $0 \leq \lambda \leq 1$ for $0 \leq t \leq t_{f}$. Here $t_{f}$ is the travel time. In closed form, position, velocity and acceleration for a straight-line trajectory can be given as, 


$$
\begin{gathered}
P=P_{0}+\lambda(t)\left[P_{f}-P_{0}\right] \\
\dot{P}=\dot{\lambda}(t)\left[P_{f}-P_{0}\right] \\
\ddot{P}=\ddot{\ddot{\lambda}}(t)\left[P_{f}-P_{0}\right]
\end{gathered}
$$

Trajectory functions used for total energy calculations are cubic segment, bang-bang parabolic blend, simple harmonic, 3-4-5 polynomial and cycloid.

\subsection{Cubic Segment Trajectory}

One of the most frequently used trajectory functions in robotics is a cubic polynomial function, which is given as,

$$
P(t)=P_{0}+\left(\frac{3 t^{2}}{t_{f}^{2}}-\frac{2 t^{3}}{t_{f}^{3}}\right)\left(P_{f}-P_{0}\right)
$$

\subsection{Bang-Bang Parabolic Blend Trajectory}

The bang-bang parabolic blend trajectory is also used in robotics applications, which consists of two parabolas. One is for $0 \leq \mathrm{t} \leq \frac{t_{f}}{2}$ and the second one is for $\frac{t_{f}}{2}<\mathrm{t} \leq \mathrm{t}_{\mathrm{f}}$. For the first part of the trajectory function

$$
P(t)=P_{0}+\frac{2 t^{2}}{t_{f}^{2}}\left(P_{f}-P_{0}\right), \quad 0 \leq t \leq \frac{t_{f}}{2}
$$

For the second part

$$
P(t)=P_{f}+\left(\frac{4 t}{t_{f}}-\frac{2 t^{2}}{t_{f}^{2}}-2\right)\left(P_{f}-P_{0}\right), \quad \frac{t_{f}}{2}<t \leq t_{f}
$$

\subsection{Simple Harmonic Trajectory}

Simple harmonic trajectory function is a trigonometric function. This and the following functions are mostly used in cam design. The simple harmonic trajectory function is given as, 


$$
P(t)=P_{0}+\frac{1}{2}\left(1-\cos \frac{\pi t}{t_{f}}\right)\left(P_{f}-P_{0}\right), \quad 0 \leq t \leq t_{f}
$$

\subsection{Cycloidal Trajectory}

The equation of the cycloidal trajectory function is given as,

$$
P(t)=P_{0}+\left(\frac{t}{t_{f}}-\frac{1}{2 \pi} \sin \frac{2 \pi t}{t_{f}}\right)\left(P_{f}-P_{0}\right), \quad 0 \leq t \leq t_{f}
$$

\subsection{3-4-5 Polynomial Trajectory}

The 3-4-5 polynomial trajectory function is given as,

$$
P(t)=P_{0}+\left(10 \frac{t^{3}}{t_{f}^{3}}-15 \frac{t^{4}}{t_{f}^{4}}+6 \frac{t^{5}}{t_{f}^{5}}\right)\left(P_{f}-p_{0}\right) \quad 0 \leq t \leq t_{f}
$$

Figure 2 shows the cycloidal trajectory, along with its velocity and acceleration as a sample.

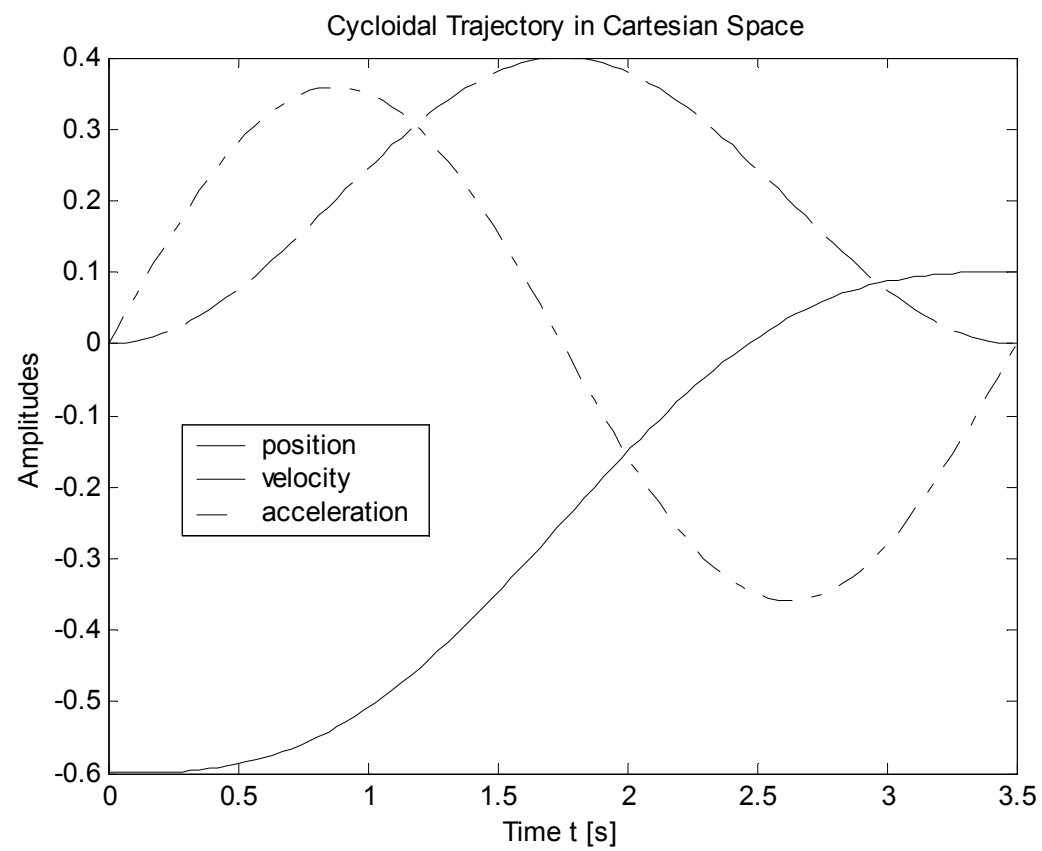

Fig. 2. Cartesian position, velocity and acceleration for cycloidal tarjectory. 


\section{Energy Calculations}

When two points in the workspace are chosen, then $P_{0}\left(x_{0}, y_{0}, z_{0}\right)$ and $P_{f}\left(x_{f}\right.$, $\left.y_{f}, z_{f}\right)$ are known. For a chosen trajectory and travel time $t_{\mathrm{f}}, \lambda(t)$ is also known. By using equation (3) any number of trajectory points $P_{i}(i=1,2, \ldots, N)$, velocity $\dot{P}_{i}$ and acceleration $\ddot{P}_{i}$ can be calculated.

When the Cartesian space position, velocity and acceleration values are computed in this manner, then the link space values, i.e., servomotor rotations, velocities and accelerations can be calculated by using the following inverse kinematic relations

$$
\begin{gathered}
\theta=T^{-1} P \\
\&=J_{p}^{-1} \dot{P} \\
\ddot{P}_{p}^{-1}-J_{p}^{-1} \dot{J}_{p} J_{p}^{-1} \dot{P}
\end{gathered}
$$

Here $\theta^{T}=\left[\theta_{1}, \theta_{2}, r\right]^{T}$ is the link rotation vector, $P^{T}=[x, y, z]^{T}$ is the Cartesian coordinate vector, $T^{-1}$ is the inverse of the transformation matrix, $J_{p}^{-1}$ is the inverse of the Jacobian matrix and $\dot{J}_{P}$ is the time derivative of the Jacobian matrix. Using the link angles, angular velocities and angular accelerations, it is possible to compute servomotor torques by the use of equation (1). Once torques and angular velocities are known, the total power requirement of the robot becomes

$$
W(t)=\sum_{q=1}^{3}\left|\tau_{q} \dot{\theta}_{q}\right|
$$

The total energy spent during the travel time $t_{f}$ is

$$
E=\int_{0}^{t_{f}} W(t) d t
$$

To serve as a practical application of the above, a series of computations were carried out and total energy consumption curves are obtained for five 
trajectories ${ }^{[2]}$. Geometrical values and dynamic parameters that are used for simulation are tabulated in Table 1.

Table 1. Geometric and dynamic properties of the spherical robot.

\begin{tabular}{|l|l|l|}
\hline$h=1.5 \mathrm{~m}$ & $m_{2}=27 \mathrm{~kg}$ & $I_{y y}^{3}=0.0291 \mathrm{kgm}^{2}$ \\
\hline$r=0.5 \mathrm{~m}$ & $m_{3}=63 \mathrm{~kg}$ & $I_{x x}^{3}=0.0291 \mathrm{kgm}^{2}$ \\
\hline$f_{\min }=0.15 \mathrm{~m}$ & $I_{z z}^{1}=0.8 \mathrm{kgm}^{2}$ & $I_{y y}^{3}=0.0134 \mathrm{kgm}^{2}$ \\
\hline$f_{\max }=0.6 \mathrm{~m}$ & $I_{x x}^{2}=0.0134 \mathrm{kgm}^{2}$ & $I_{z z}^{3}=0.0582 \mathrm{kgm}^{2}$ \\
\hline$a_{2}=0.3 \mathrm{~m}$ & $I_{y y}^{2}=0.0537 \mathrm{kgm}^{2}$ & $m_{p}=5 \mathrm{~kg}$ \\
\hline$a_{3}=0.75 \mathrm{~m}$ & $I_{z z}^{2}=0.0134 \mathrm{kgm}^{2}$ & \\
\hline
\end{tabular}

The end point of the manipulator will travel from $\mathrm{P}_{0}(0.6,-0.6,0.5) \mathrm{m}$ to $\mathrm{P}_{\mathrm{f}}$ $(0.5,0.1,0.6) \mathrm{m}$ during a travel time $\mathrm{t}_{\mathrm{f}}=3.5 \mathrm{~s}$. Figures 3,4 and 5 show angular positions, angular accelerations and angular velocities of the links of the manipulator for the cycloidal trajectory, respectively. Figure 6 shows the torque requirements of servomotors during the travel. Figure 7 shows the power needed for each servomotor. Figure 8 is the plot of the total power requirement of the robot. The integral of the curve, which is given in Figure 7 will yield the total energy required for the manipulator, which is 66.63 Joule. If these calculations are repeated for different travel times $t_{f}$, the plot of the total consumed energy per travel time can be obtained. The result of a such set of computations for the cycloidal trajectory function is summarized in Figure 9.

Total energy consumption curves can be obtained for the other four trajectories as well in a similar manner.The plot of the total consumed energy curves for five trajectories are shown in Figure 10. Cyloid and bang-bang parabolic blend trajectories are spending almost the same amount of energy. For longer travel times, curves are approaching to an asymptotic value. This is because, the effect of inertial forces are decreasing and becoming negligible for longer travel times, servomotors are overcoming only gravitational forces and moments. In terms of energy consumption cubic segment is the best choice. Since 3-4-5 polynomial trajectory and cycloidal trajectory functions are starting and ending with zero accelerations, they can be preferred to eliminate vibrations and for the smooth running of the robot. 


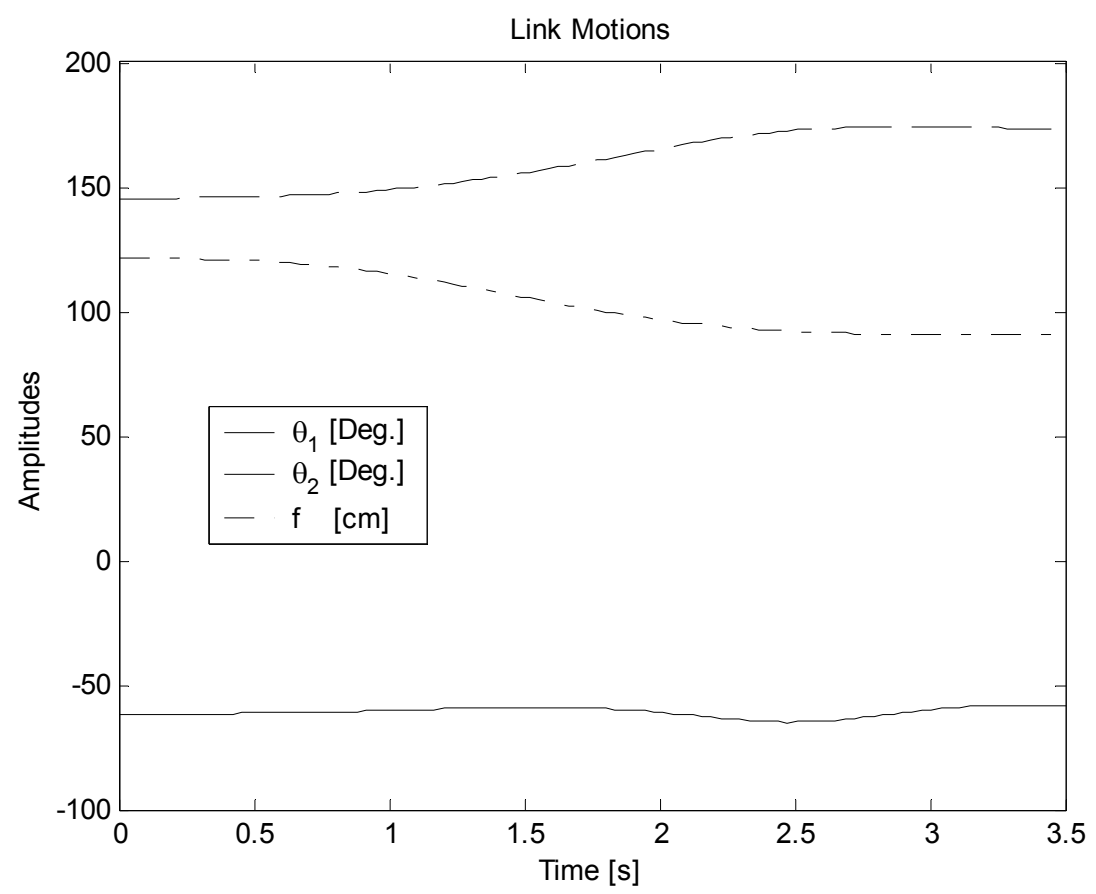

Fig. 3. Link angles for cycloidal trajectory.

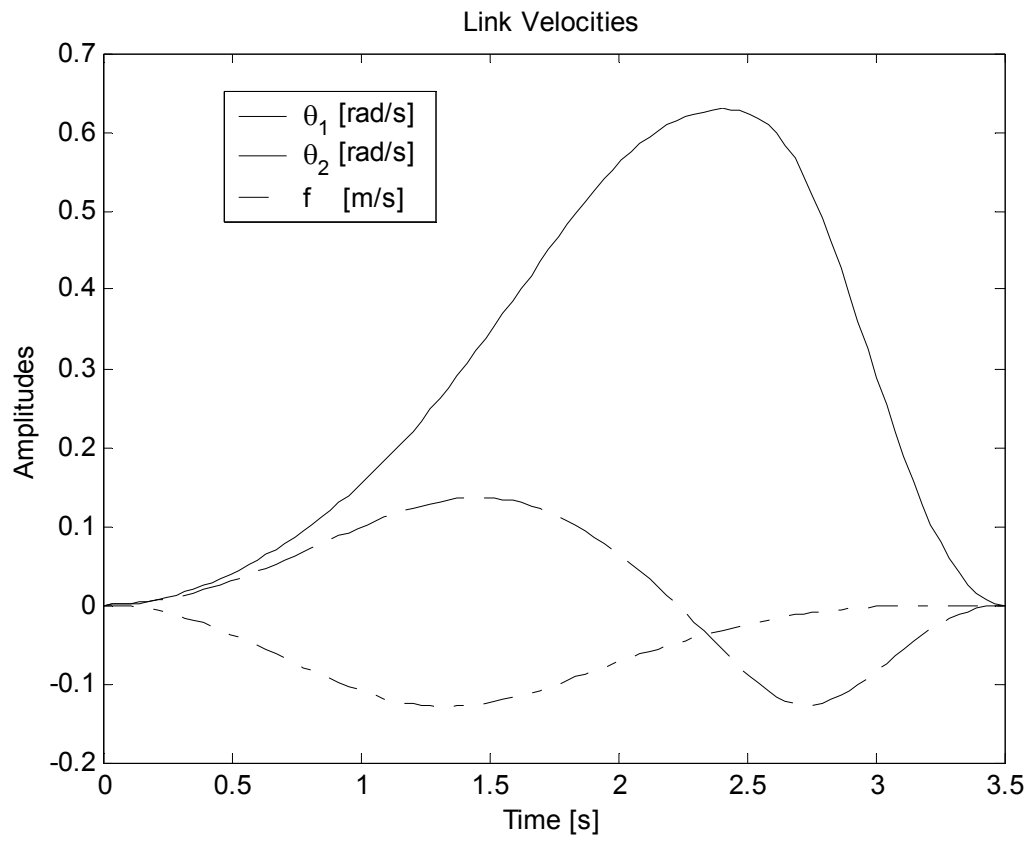

Fig. 4. Link angular velocities for cycloidal trajectory. 


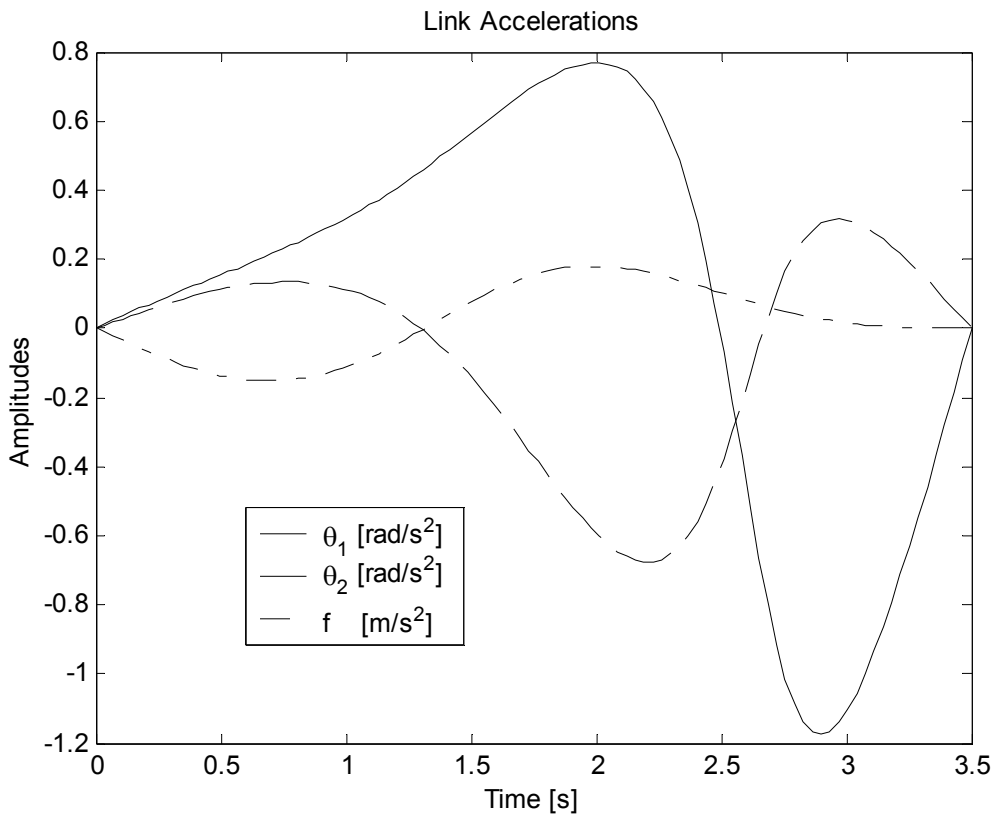

Fig. 5. Link angular accelerations for cycloidal trajectory.

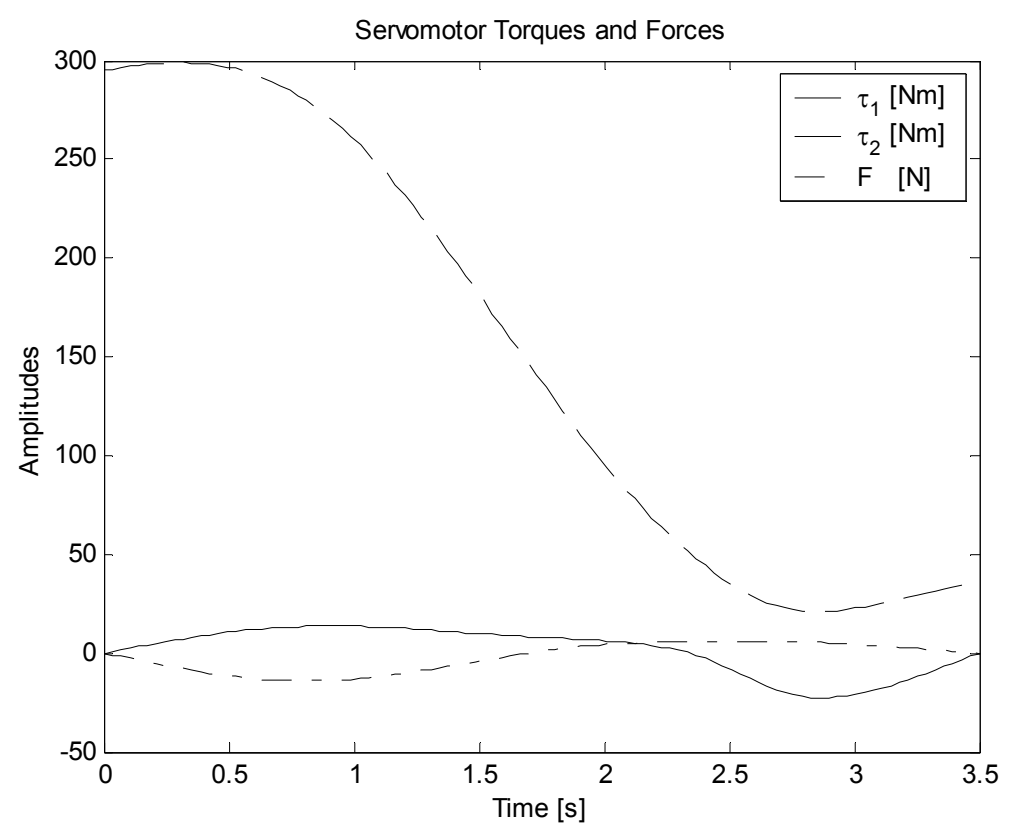

Fig. 6. Motor torques of the manipulator. 


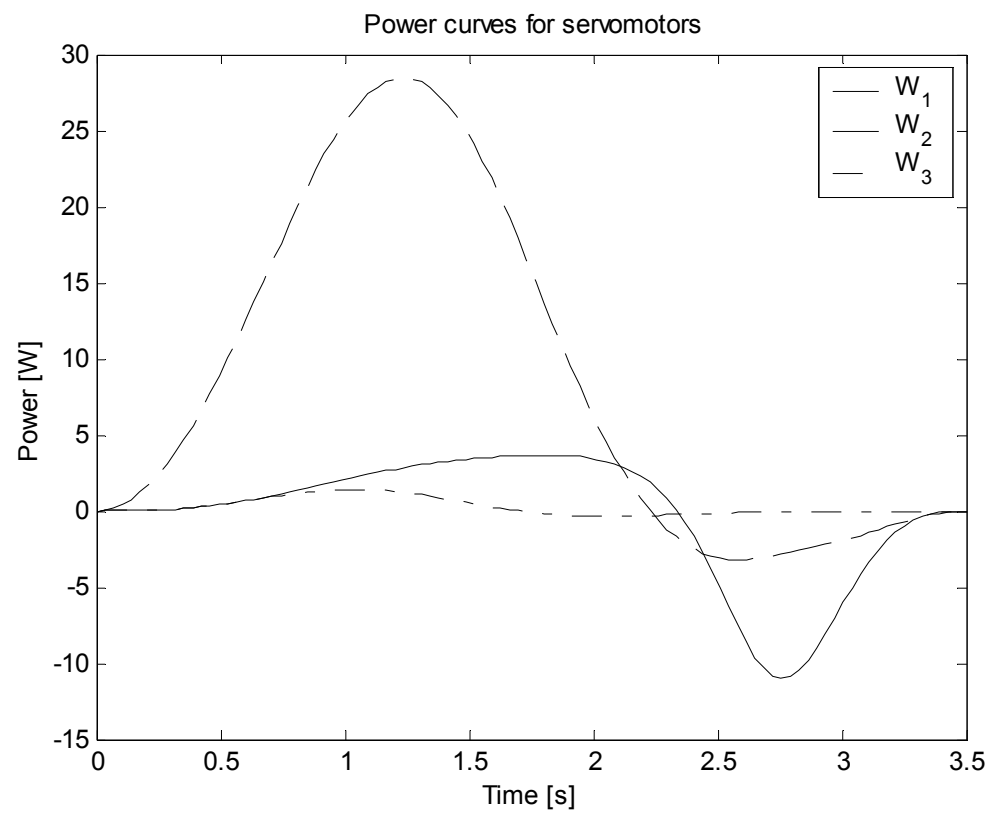

Fig. 7. Servomotor power consumption curves.

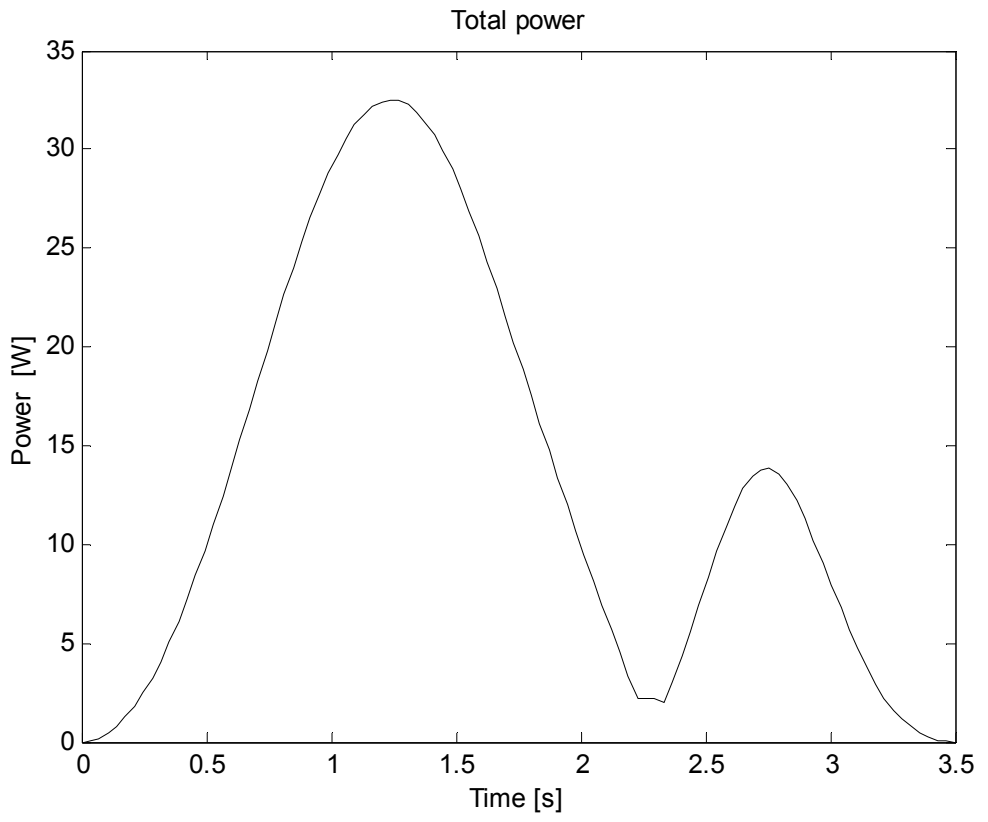

Fig. 8. Total power required for the manipulator. 


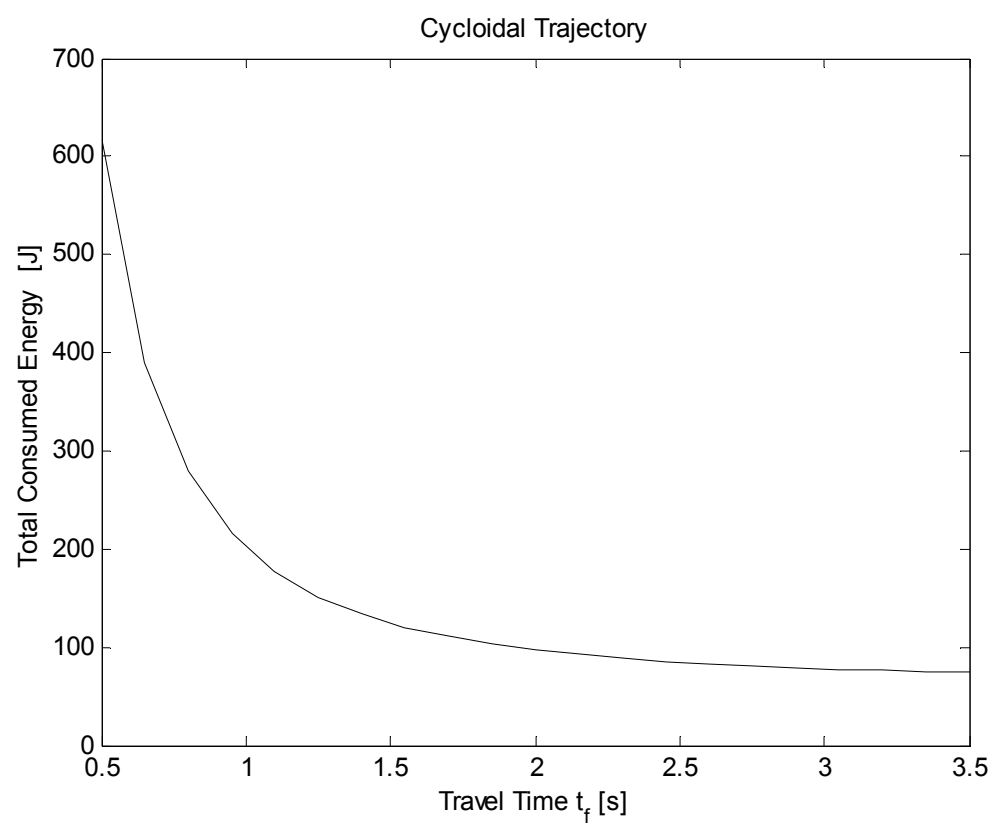

Fig. 9. Total consumed energy curve for cycloidal trajectory.

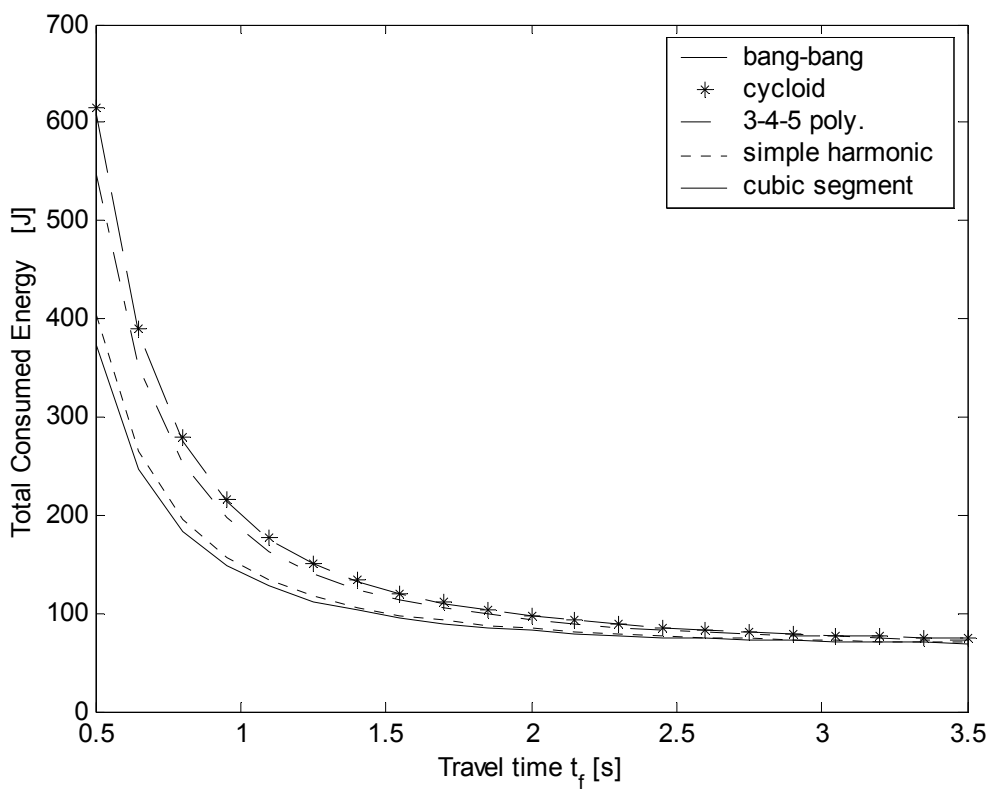

Fig. 10. Total consumed energy curves for all trajectories. 


\section{Conclusion}

In this study five different trajectory functions are used for the travel of the end effector of a Stanford type spherical manipulator to calculate total consumed energy. These trajectories are namely; cycloid, 3-4-5 polynomial, bang-bang parabolic blend, simple harmonic and cubic segment. Minimum energy is spent by the cubic segment trajectory. For the cycloidal and 3-4-5 polynomial trajectories, acceleration curve is continuous and zero at the beginning and at the end, jerk is eliminated and smooth running is possible. Cycloidal and 3-4-5 polynomial trajectories should be preferred to others, if the energy consumption is not the prime concern.

\section{References}

[1] Wolovich, W. A., (1987), Robotics: Basic Analysis and Design, Holt, Rinehart and Winston Inc., USA.

[2] Shahrani, S. A., January (2002), "Optimum Trajectory Calculations for the Minimum Energy Requirements of a Spherical Robot by Symbolic Programming," M. Sc. Thesis, Production Engineering and Mechanical Systems Design Department, King Abdulaziz University, Saudi Arabia. 


\title{
لختيار معادلة المسار للحصول على ألزطلة مطلوبة لروبوت كروي لمبحول
}

\author{
أ.أن. الجاوي، ح ـ دليكن ،ش . الشهراني

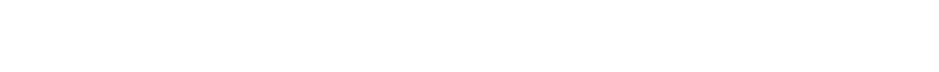

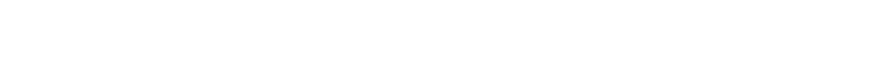

المستخلص: في هذه الدرلسة مم قطوير المعادلات الدينلميكية لروبوت

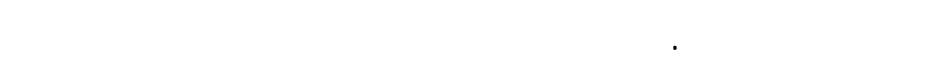

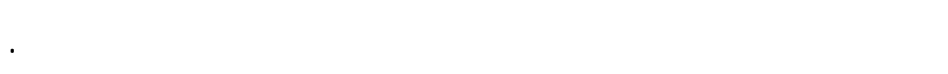

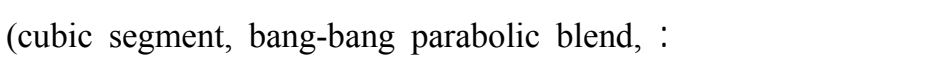
( simple harmonic, cycloid, and 3-4-5 polynomial)

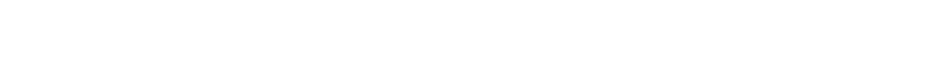

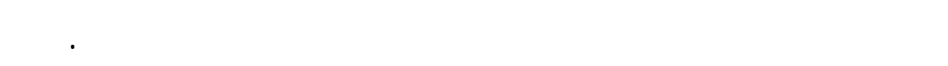

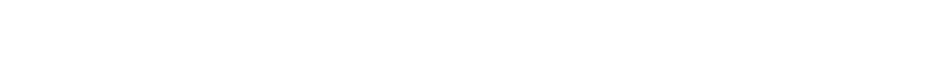

